\title{
Impact of Harmonics on the Interpolated DFT Frequency Estimator
}

\author{
Daniel Belega ${ }^{1}$, Dario Petri ${ }^{2}$, and Dominique Dallet ${ }^{3}$
}

${ }^{1}$ Department of Measurements and Optical Electronics, Politehnica University Timisoara, Bv. V. Parvan, Nr. 2, 300223, Timisoara, Romania, Phone: +40 25640 33 65, Fax: +40 2564033 62, E-mail: daniel.belega@upt.ro

${ }^{2}$ Department of Industrial Engineering, University of Trento, Via Sommarive, 14-38100, Trento, Italy, Phone: +39 0461 883902, Fax: +39 0461 882093, E-mail: dario.petri@unitn.it

${ }^{3}$ IMS Laboratory, University of Bordeaux, Bordeaux INP, CNRS UMR5218, 351 Cours de la Libération, Bâtiment A31, 33405, Talence Cedex, France, Phone : +33 5400026 32, Fax : +33 55637 15 45, E-mail: dominique.dallet@ims-bordeaux.fr

Abstract - The paper investigates the effect of the interference due to spectral leakage on the frequency estimates returned by the Interpolated Discrete Fourier Transform (IpDFT) method based on the Maximum Sidelobe Decay (MSD) windows when harmonically distorted sine-waves are analyzed. The expressions for the frequency estimation error due to both the image of the fundamental tone and harmonics, and the frequency estimator variance due to the combined effect of both the above disturbances and wideband noise are derived. The achieved expressions allow us to identify which harmonics significantly contribute to frequency estimation uncertainty. A new IpDFT-based procedure capable to compensate all the significant effects of harmonics on the frequency estimation accuracy is then proposed. The derived theoretical results are verified through computer simulations. Moreover, the accuracy of the proposed procedure is compared with those of other state-of-the-art frequency estimation methods by means of both computer simulations and experimental results. 
Keywords: Error and uncertainty analysis, frequency estimation, interpolated DFT method, spectral interference, windowing.

\section{Introduction}

Accurate estimates of harmonically distorted sine-waves are needed in many engineering applications such as instrumentation, vibration analysis, power systems, and communications. To estimate the parameters of a multi-frequency signal, Discrete Fourier Transform (DFT)-based methods are usually preferred to the parametric ones (e.g. autoregressive methods, Pisarenko's algorithm, MUSIC algorithm, ESPRIT algorithm, sine-fitting algorithm [1-3]) since they provide accurate estimates, require a smaller processing effort, and are more robust to signal model uncertainties. Many DFT-based methods have been proposed in the literature, such as the Interpolated DFT (IpDFT) method [3-10], the phase difference methods [11-13], the least squares-based methods [14-15], and the energy-based method [16-17].

The IpDFT method is widely adopted to compensate the picket-fence effect due to the intrinsic frequency granularity of the DFT spectrum [8]. In particular, when a Maximum Sidelobe Decay (MSD) window is used to reduce spectral leakage $[9,18]$ the multi-frequency signal parameters can be estimated by means of simple analytical relationships [9]. By using the IpDFT method, the frequency of each spectral tone is estimated by interpolating the two largest DFT samples belonging to the corresponding spectrum peak. The related amplitude and phase parameters are then estimated by using the obtained frequency value. When analysing harmonically distorted sine-waves, IpDFT frequency estimates can be affected by spectral leakage from the image components, harmonics, and wideband noise [3, 7, 10]. In particular, in some applications, such as vibration analysis of low-speed rotating machines, the analysed signals contain significant harmonic components [3, 4], which may heavily affect the frequency estimation accuracy. The contribution of the harmonics to frequency estimation uncertainty has been analysed in [10], where the magnitude of the related frequency estimation error has been derived. In order to further reduce of the above detrimental effects, the multi-point IpDFT methods have been proposed [19-21]. They hinder the effect of spectral leakage from both the image of the fundamental component and harmonics by 
weighted the interpolated DFT samples using coefficients related to classical finite difference procedures $[19,20]$. A corrected IpDFT algorithm has been also proposed for that aim [22, 23]. That procedure estimates the sine-wave frequency by subtracting to the interpolated DFT samples the contributions due to the image components and harmonics. However, to the best of the authors' knowledge, expressions for the contribution of harmonics and wideband noise on the frequency estimator accuracy when the IpDFT approach is used has not yet published in the scientific literature.

The aim of this paper is twofold. At first the effect of the spectral leakage of both the fundamental image component, harmonics, and wideband noise on the IpDFT frequency estimator based on a MSD window are determined. The obtained expressions are then employed to propose a new IpDFT-based procedure capable to reject interference from other tones on frequency estimation accuracy.

The remaining of the paper is organized as follows. In Section 2 the expressions for the frequency estimation error due to both the fundamental image component and harmonics and the combined frequency estimator variance due to the above disturbance components and wideband noise are derived when the MSD windows are adopted. An IpDFT-based frequency estimation procedure that compensates the effect of spectral leakage from the fundamental image and significant harmonics is then proposed in Section 3. In Section 4 the accuracy of the provided expressions is verified through computer simulations. Moreover, the proposed procedure is compared with the classical IpDFT method, the corrected IpDFT (IpDFTc) procedure [22, 23], and the multi-harmonic sine-fitting (MHSF) method [24] by means of both computer simulations and experimental results. Finally, Section 5 concludes the paper.

\section{Analytical expression of the frequency estimation error}

Let us consider the following discrete-time noisy and harmonically distorted sine-wave of length $M$, composed by $K$ harmonics and sampled at frequency $f_{s}$ :

$$
\begin{aligned}
x(m)=A_{1} \sin \left(2 \pi f\left(m-\frac{M-1}{2}\right)+\varphi_{1}\right)+\sum_{k=2}^{K} A_{k} \sin \left(2 \pi k f\left(m-\frac{M-1}{2}\right)+\varphi_{k}\right) & +e(m), \\
& m=0,1,2, \ldots, M-1
\end{aligned}
$$


where $A_{k}$ and $\varphi_{k}(k=1,2, \ldots, K)$ are respectively the amplitude and the phase of the $k$ th spectral line, $f$ is the signal normalized or discrete frequency, defined as the ratio $f=f_{\text {in }} / f_{s}$ between the continuous-time signal frequency $f_{\text {in }}$ and the sampling frequency $f_{s}$, and $e(\cdot)$ is an additive white Gaussian noise of zero mean and variance $\sigma_{n}^{2}$. It is worth noticing that the time reference has been assumed at the centre of the observation interval (i.e. at time $(M-1) / 2)$ in order to minimize the effect of wideband noise on the estimated phase [7].

The discrete frequency $f$ can be written as:

$$
f=\frac{f_{\text {in }}}{f_{s}}=\frac{v}{M}=\frac{l+\delta}{M}
$$

where $v$ represents the number of observed sine-wave cycles or the normalized frequency expressed in spectral bins, $l$ is its rounded value and $\delta(-0.5 \leq \delta<0.5)$ is the difference between the above values.

The sampling process can be either coherent (i.e. $\delta=0$ ) or non-coherent (when $\delta \neq 0$ ) [25]. The latter case is often encountered in practice due to the lack of synchronization between the analysed continuous-time waveform and the sampling rate. When non-coherent sampling occurs the discrete spectrum of the signal (1) is affected by spectral leakage, which can be reduced by windowing, i.e. by multiplying the acquired signal (1) by a suitable window sequence $w(m), m=0,1, \ldots, M-1$ [26], so obtaining the windowed signal $x_{w}(m)=x(m) \cdot w(m), m=0,1, \ldots, M-1$. Cosine-class windows are often adopted, that is [27]:

$$
w(m)=\sum_{h=0}^{H-1} a_{h} \cos \left(2 \pi \frac{h}{M}\left(m-\frac{M-1}{2}\right)\right), \quad m=0,1, \ldots, M-1
$$

where $H(H \geq 2)$ represents the number of window terms and $a_{h}>0, h=0,1, \ldots, H-1$, are the window coefficients.

The Discrete-Time Fourier Transform (DTFT) of the windowed signal $x_{w}(\cdot)$ is defined as [27]:

$$
X_{w}(\lambda)=\sum_{m=0}^{M-1} x_{w}(m) e^{-j 2 \pi \frac{\lambda}{M}\left(m-\frac{M-1}{2}\right)}+E_{w}(\lambda), \quad \lambda \in[0, M)
$$


where $\lambda$ is the normalized frequency, expressed in spectral bins, and $E_{w}(\cdot)$ is the DTFT of the signal $e(m) \cdot w(m)$.

By neglecting the contribution of wideband noise, and using (1), (3), and (4), the following expression can be achieved for DFT samples at the integer values $r$ of the normalized frequency close to $v$.

$$
X_{w}(r) \cong \frac{A_{1}}{2 j} W(r-v) e^{j \varphi_{1}}-\frac{A_{1}}{2 j} W(r+v) e^{-j \varphi_{1}}+\sum_{k=2}^{K} \frac{A_{k}}{2 j} W(r-k v) e^{j \varphi_{k}}, \quad r \approx v
$$

The second term in (5) represents the image of the fundamental component while the contribution of the image components of harmonics has been omitted since simulations showed it is negligible as compared with the spectral components considered in (5).

In (5), $W(\cdot)$ represents the DTFT of the adopted window $w(\cdot)$, which for $|\lambda|<<M$ can be expressed as [28]:

$$
W(\lambda) \cong \frac{M \sin (\pi \lambda)}{\pi} \sum_{h=0}^{H-1}(-1)^{h} a_{h} \frac{\lambda}{\lambda^{2}-h^{2}} \quad \text { when }|\lambda|<<M \text {. }
$$

It is worth observing that, since the time reference is located in the centre of the observation interval, (6) represents a real-valued and even function, i.e. $W(-\lambda)=W(\lambda)$.

In the particular case of the $H$-term MSD window $(H \geq 2)$ the coefficients $a_{h}$ are [9]:

$$
a_{0}=\frac{C_{2 H-1}^{H-1}}{2^{2 H-2}} \text { and } a_{h}=\frac{C_{2 H-2}^{H-h-1}}{2^{2 H-3}}, \quad h=1,2, \ldots H-1
$$

where $C_{p}^{q}=\frac{p !}{(p-q) ! q !}$. The related DTFT (6) can be expressed as [9]:

$$
W(\lambda) \cong \frac{M \sin (\pi \lambda)}{2^{2 H-2} \pi \lambda} \frac{(2 H-2) !}{\prod_{h=1}^{H-1}\left(h^{2}-\lambda^{2}\right)}, \quad|\lambda|<<M
$$

When the signal-to-noise ratio in the frequency domain (equal to the signal-to-noise-ratio in the time domain multiplied by the number of analyzed samples) is higher than about $18 \mathrm{~dB}$, the integer part $l$ of the number of observed signal cycles can be exactly determined with high probability by simply applying a maximum search procedure to the DFT samples $\left|X_{w}(r)\right|, r=0,1, . ., M / 2-1$ [29], [30]. 
109 determining the ratio:

$$
\alpha=\frac{\left|X_{w}(l+s)\right|}{\left|X_{w}(l-1+s)\right|}
$$

$110 \quad$ where $s=0$ if $\left|X_{w}(l-1)\right|>\left|X_{w}(l+1)\right|$ and $s=1$ if $\left|X_{w}(l-1)\right|<\left|X_{w}(l+1)\right|$.

111 By considering negligible the contributions of the image component and harmonics in (5) are negligible, it

112 follows that:

$$
\alpha \cong \frac{W(-\delta+s)}{W(-1-\delta+s)}
$$

113 which, using (8), for the $H$-term MSD window becomes:

$$
\hat{\delta}=\frac{(H-1+s) \alpha-H+s}{\alpha+1} .
$$

114 In the following the effect of the fundamental image component, harmonics and wideband noise on

115 the frequency estimator provided by the IpDFT method based on the MSD windows are separately 116 analyzed in the case of harmonically distorted sine-waves or noisy and harmonically distorted sine-waves, 117 respectively.

118 a) harmonically distorted sine-waves

119 Due to the spectral interference from both the fundamental image component and harmonics (whose 120 effect has been neglected in (11)), the returned values are affected by an error (see (A.16) in the 121 Appendix):

$$
\Delta \delta \cong \Delta \delta_{0}+\sum_{k=2}^{K} \Delta \delta_{k}
$$

122 where:

$$
\Delta \delta_{0}=\frac{2(-1)^{s+1}\left(H+(-1)^{s} \delta\right)(l+\delta)}{2 l+\delta+(-1)^{s+1} H} \frac{W(2 l+\delta)}{W(\delta)} \cos \left(2 \varphi_{1}\right),
$$

123 represents the contribution of the fundamental image component, while: 


$$
\Delta \delta_{k}=(-1)^{s}(k-1) \frac{A_{k}}{A_{1}} \frac{\left(H+(-1)^{s} \delta\right)(l+\delta)}{(k-1) l+k \delta+(-1)^{s} H} \frac{W((k-1) l+k \delta)}{W(\delta)} \cos \left(\varphi_{k}-\varphi_{1}\right), \quad k=2,3, \ldots, K
$$

124 is the contribution of the $k$-th harmonic.

125 It is worth noticing that:

126 - the errors $\Delta \delta_{0}$ and $\Delta \delta_{k}$ are sinusoidal functions of the related tone phase; the corresponding maximum

$127 \quad$ value are respectively:

$$
\Delta \delta_{0 \max }=\frac{2\left(H+(-1)^{s} \delta\right)(l+\delta)}{2 l+\delta+(-1)^{s+1} H} \frac{W(2 l+\delta)}{W(\delta)},
$$

128 and

$$
\Delta \delta_{k \max }=(k-1) \frac{A_{k}}{A_{1}} \frac{\left(H+(-1)^{s} \delta\right)(l+\delta)}{(k-1) l+k \delta+(-1)^{s} H} \frac{W((k-1) l+k \delta)}{W(\delta)}, \quad k=2,3, \ldots, K
$$

- the error $\Delta \delta_{k}$ depends on $\delta$ and it is null when coherent sampling occurs (since the related value of the window transform is null) or the phase difference $\varphi_{k}-\varphi_{1}= \pm \pi / 2$ rad;

- the error $\Delta \delta_{k}$ increases as the amplitude $A_{k}$ increases, $l$ decreases, or $k$ decreases.

\section{b) noisy and harmonically distorted sine-waves}

To evaluate the contribution of noise $e(\cdot)$, the number of acquired signal cycles $v$ is assumed high enough that the contributions of the fundamental image component and harmonics can be neglected. In this case the variance of the estimator $\hat{\delta}$ due wideband noise is given by [9]:

$$
\sigma_{\hat{\delta}, n}^{2} \cong \frac{(H-|\delta|)^{2}\left[2(4 H-3)\left(\delta^{2}-|\delta|\right)+2 H^{2}-1\right]}{(2 H-1)^{3}} \frac{E N B W}{S L^{2}(\delta)} \frac{1}{M \cdot S N R},
$$

where ENBW and $S L(\delta)$ are the Equivalent Noise BandWidth and the Scalloping Loss of the adopted window, given by [9]:

$$
E N B W=\frac{C_{4 H-4}^{2 H-2}}{\left(C_{2 H-2}^{H-1}\right)^{2}} \text {, and } S L(\delta)=\frac{\sin (\pi \delta)}{\pi \delta} \frac{[(H-1) !]^{2}}{\prod_{h=1}^{H-1}\left(h^{2}-\delta^{2}\right)}
$$


and $S N R=A^{2} /\left(2 \sigma_{n}^{2}\right)$ is the Signal-to-Noise Ratio.

Since noise and signal components are related to different physical phenomena, their effects can be considered statistical independent. Thus, by considering the phases of the fundamental component and harmonics uniformly distributed in the range $[0,2 \pi) \mathrm{rad}$, the combined variance of the estimator $\hat{\delta}$ is [31]:

$$
\sigma_{\hat{\delta}}^{2}=\rho_{0}+\sum_{k=2}^{K} \rho_{k}+\sigma_{\hat{\delta}, n}^{2}=0.5\left(\Delta \delta_{0 \max }\right)^{2}+0.5 \sum_{k=2}^{K}\left(\Delta \delta_{k \max }\right)^{2}+\sigma_{\hat{\delta}, n}^{2},
$$

where $\rho_{0}=\Delta \delta_{0 \max }^{2} / 2$ and $\rho_{k}=\Delta \delta_{k, \max }^{2} / 2, k=2,3, \ldots, K$ are the variances of the contributions of the fundamental component and the $k$ th harmonic to the frequency estimation error, and $\sigma_{\hat{\delta}, n}^{2}$ is given by (17).

From (18) it follows that the contribution of spectral interference from the fundamental image component and harmonics becomes negligible as compared with the effect of wideband noise when:

$$
S N R<<\frac{(H-|\delta|)^{2}\left[2(4 H-3)\left(\delta^{2}-|\delta|\right)+2 H^{2}-1\right]}{(2 H-1)^{3} M} \frac{E N B W}{S L^{2}(\delta)} \frac{1}{\rho_{0}+\sum_{k=2}^{K} \rho_{k}} .
$$

\section{The proposed IpDFT-based procedure}

Since the sidelobe envelope of the $H$-term MSD window spectrum has a fast decaying rate (i.e. $6(2 H$ 1) $\mathrm{dB}$ /oct), only the lower order harmonics are expected to provide a significant contribution to $\Delta \delta$ and $\sigma_{\hat{\delta}}^{2}$. Fig. 1 shows the theoretical expressions for the maximum errors $\Delta \delta_{2 \max }, \Delta \delta_{3 \max }$, and $\Delta \delta_{4 \max }$ normalized to $A_{2} / A_{1}, A_{3} / A_{1}$, and $A_{4} / A_{1}$, respectively as a function of $\delta$ when $l=3$ (Fig. $\left.1(\mathrm{a})\right)$ and $l=$ 10 (Fig. 1(b)). The term $\delta$ is varied in the range $(-0.5,0.5)$ with a step of 0.04 cycles and the Hann window is adopted. 


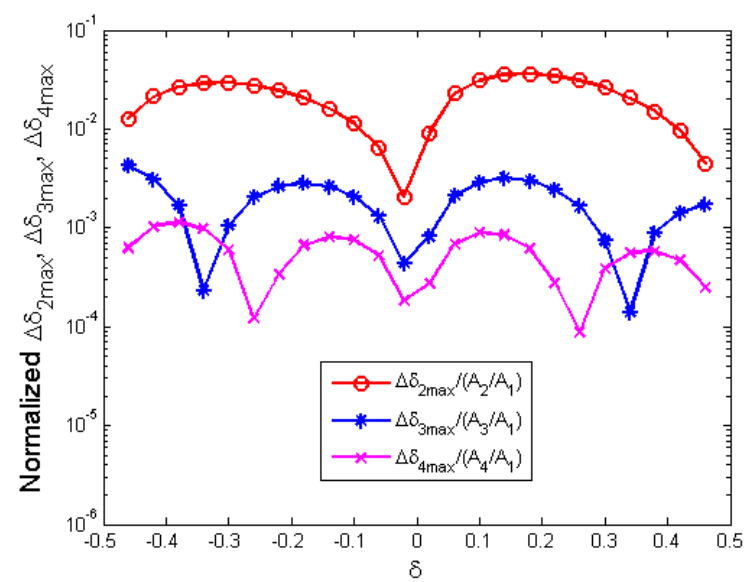

(a)

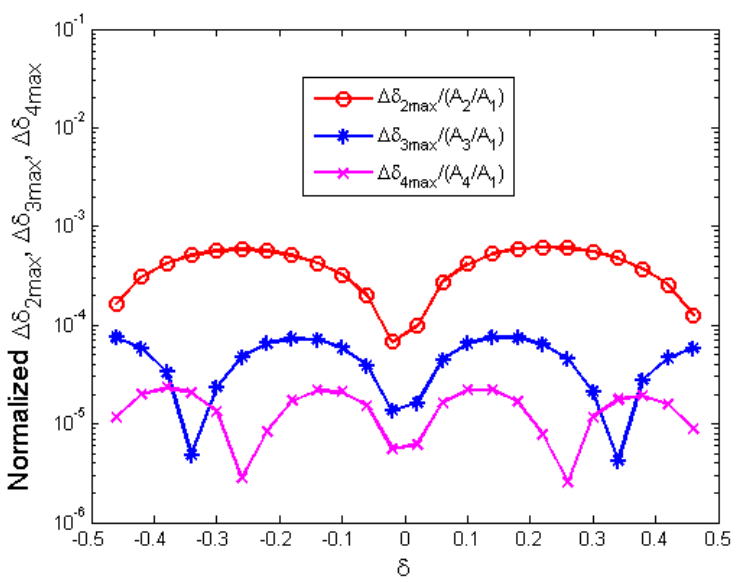

(b)

Fig. 1. Theoretical expressions for the maximum errors $\Delta \delta_{2 \max }, \Delta \delta_{3 \max }$, and $\Delta \delta_{4 \max }$ normalized to $A_{1} / A_{2}$, $A_{1} / A_{3}$, and $A_{1} / A_{4}$, respectively when (a) $l=3$ and (b) $l=10$. The Hann window is adopted.

Fig. 1 shows that, for both values of $l$, the contribution of the 2nd harmonic always dominates the others when equal amplitude harmonics are considered. Also, the contribution of the 3rd harmonic overcome that of the 4th harmonic for most values of $\delta$. Moreover, by comparing Figs. 1(a) and (b), it follows that the harmonic contributions quickly decreases as $l$ increases. This phenomenon is due to the rapidly decaying sidelobe level of the Hann window spectrum.

Leveraging on the results derived in the previous Section the frequency estimation procedure described in the following using pseudocode is proposed:

1) Acquire $M$ samples of the analyzed signal $x(m), m=0,1, \ldots, M-1$.

2) Evaluate the windowed signal $x_{w}(m)=x(m) w(m), m=0,1, \ldots, M-1$, where $w(\cdot)$ is the H-term MSD window.

3) Evaluate the DFT of the windowed signal $x_{w}(\cdot)$ by (4).

4) Apply a maximum search procedure to the DFT samples to determine the integer part l of the number of observed cycles.

5) Apply the IpDFT method to determine the estimates for the term $\hat{\delta}_{x}$, the amplitudes and the phases of the fundamental and the harmonics, $\hat{A}_{1 x}, \hat{\varphi}_{1 x}$ and $\hat{A}_{k x}, \hat{\varphi}_{k x}, k=2,3, \ldots$

6) Apply (14) to determine the contribution of each harmonic to the estimation error $\Delta \delta_{k y}, k=2,3, \ldots$.

7) Identify the harmonic orders $p_{x}$ and $q_{x}$ related to the two errors with greatest magnitude

8) Determine $\hat{v}_{x}=I+\hat{\delta}_{x}-\Delta \hat{\delta}_{0_{x}}-\Delta \hat{\delta}_{p_{x}}-\Delta \hat{\delta}_{q_{x}}$ 
9) Remove the estimates of the $p_{x}$-th and $q_{x}$-th harmonics from the signal

$$
y(m)=x(m)-\hat{A}_{p_{x}} \sin \left(2 \pi p_{x} \frac{\hat{v}_{x}}{M} m+\hat{\varphi}_{p_{x}}\right)-\hat{A}_{q_{x}} \sin \left(2 \pi q_{x} \frac{\hat{v}_{x}}{M} m+\hat{\varphi}_{q_{x}}\right), \quad m=0,1,2, \ldots, M-1
$$

10) Perform steps 5) and 6) on signal $y(\cdot)$ and estimate the term $\delta$ by the following expression:

$$
\widetilde{\delta}=\hat{\delta}_{y}-\Delta \hat{\delta}_{0_{y}}-\sum_{k \in K_{h}} \Delta \hat{\delta}_{k_{y}}
$$

where $\hat{\delta}_{y}$ is the estimator of $\delta$ obtained by applying the IpDFT method to the signal $y(\cdot), \Delta \hat{\delta}_{0_{y}}$ and $\Delta \hat{\delta}_{k_{y}}$ are determined by (13) and (14), respectively, using the harmonic parameters returned by the IpDFT method and $K_{h}$ is the set containing the orders of the significant harmonics; the kth harmonic is considered significant if $\left|\Delta \hat{\delta}_{k_{y}}\right| \geq\left|\Delta \hat{\delta}_{p_{y}}\right| / \mu$ (e.g. $\mu=10$ or greater), otherwise its contribution is assumed negligible; in the previous equation $p_{y}$ is the order of the harmonic with the greatest contribution to the estimation error related to the application of the IpDFT method to the signal $y$.

As it can be seen the proposed procedure requires to perform two iterations. The aim of each iteration is to reduce a fraction of harmonics contribution on the estimation of the term $\delta$. During the first iteration (steps 1-8) the two harmonics with the greatest contribution to the estimation error are determined, their parameters are estimated through the IpDFT method, and the estimate of $\delta$ corrected from the contribution of these two harmonics is determined. Then, these contributions are removed from the original signal (step 9), so rejecting the spectral interference from both the harmonics and their images on the estimated value of $\delta$. It is worth noticing that the two harmonics providing the greatest contribution to the estimation error have been considered in the procedure in order to achieve very accurate results already after a single iteration also when the signal is effected by one harmonic of significant amplitude and one harmonic with lower amplitude, but closely spaced in frequency to the fundamental component.

In the second iteration (step 10) the proposed frequency estimation procedure is applied to the achieved signal. Further removal of harmonics contribution is no more required, since it assumes a negligible value as compared to the first iteration one; thus the application of (12)-(14) suffices. 


\section{Computer simulations and experimental results}

In this Section the accuracies of expressions (12) and (18) are firstly verified by means of computer simulations. Then, the accuracies of the proposed procedure, the classical IpDFT method [3-10], the IpDFTc procedure [22, 23], and the MHSF method [24] are compared through both computer simulations and experimental results. It is worth noticing that in [23] it has been shown that the IpDFTc procedure provides more accurate frequency estimates than the classical three- and five-point IpDFT methods [19, 20]. The MHSF method is considered in the comparison since simulations showed that it provides a minimum variance estimator when the analyzed waveform is corrupted by additive white Gaussian noise and the number of harmonics is a-priori known [29]. Conversely, when the number of harmonics is unknown the MHSF method, unlike the IpDFT methods, may provide low accuracy estimates. In addition, it requires a significantly higher processing effort than the considered IpDFT methods. These two aspects will be also analyzed in this Section.

\subsection{Simulation results}

Computer simulations were performed by considering, for each value of $v$, the fundamental tone amplitude $A_{1}=1$, phases of the fundamental and harmonics chosen at random in the range $[0,2 \pi) \mathrm{rad}$, and $M=512$ samples long data records. The two-term MSD window was adopted and a coefficient $\mu=10$ was considered in the proposed procedure. Three iterations were employed in the MHSF method since no significant accuracy improvement was achieved when using a higher number of iterations. All methods were applied to the same signal. The noise variance was chosen in such a way to achieve $S N R=50 \mathrm{~dB}$.

When considering harmonically distorted sine-waves the maximum absolute value of the frequency estimation error is adopted as accuracy parameter. Conversely, a statistical parameter, i.e. the combined standard deviation of the frequency estimator, is used when waveforms corrupted by wideband noise are considered. 
Fig. 2 shows the maximum absolute value of the frequency estimation errors, $|\Delta \delta|_{\max }$, returned by

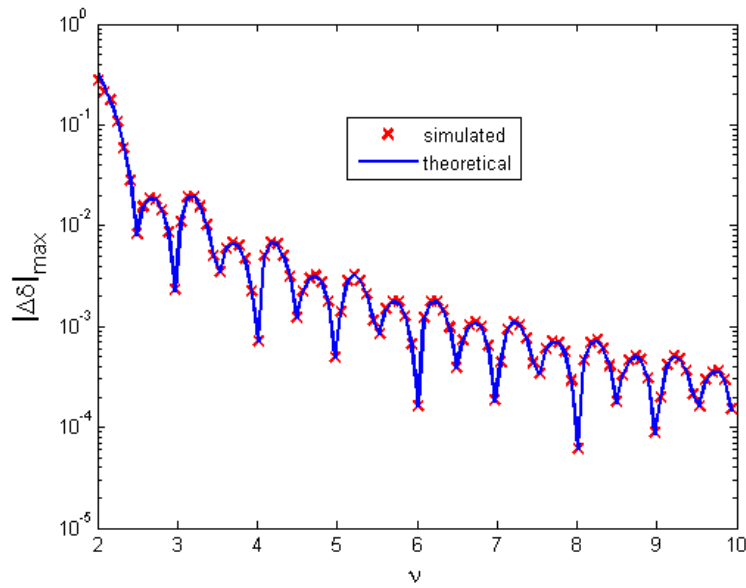

(a)

For each value of $v, 1000$ runs were performed. theoretical results.

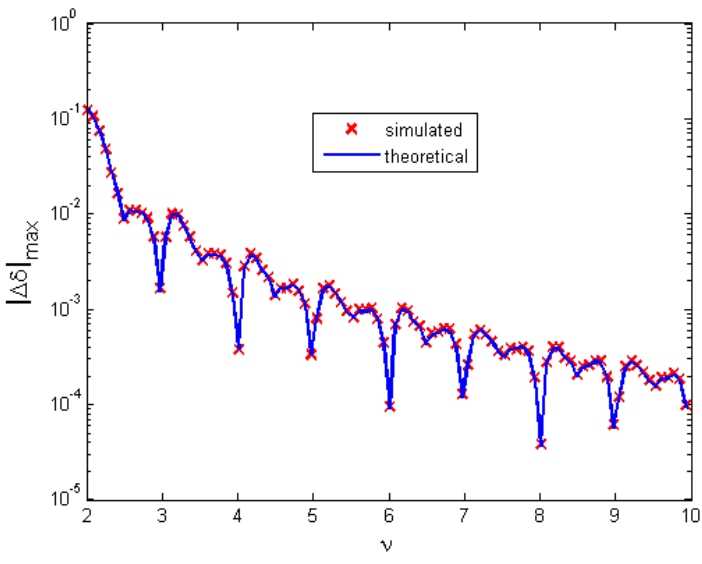

(b)

simulations and (12)-(14) as a function of $v$ for two different signals with harmonics up to the fourth order. In the first signal the 2nd harmonic prevails $\left(A_{2}=0.5, A_{3}=0.25\right.$, and $\left.A_{4}=0.125\right)$, while in the second one the 3rd harmonic overcomes the others $\left(A_{2}=0.2, A_{3}=0.6\right.$, and $\left.A_{4}=0.4\right)$. These signals were considered since situations in which the 2nd or the 3rd harmonics prevails are often encountered in practice. The normalized frequency $v$ was varied in the range $[2.01,12)$ cycles with a step of 0.04 cycles.

As we can see, for both considered signals a very good agreement is achieved between the simulation and

Fig. 2. Maximum errors $|\Delta \delta|_{\max }$ versus $v$ returned by simulations (crosses) and (12)-(14) (solid lines) when analyzing a sine-wave with amplitude $A_{1}=1$ corrupted by 2nd, 3rd, and 4th harmonics with amplitudes (a) $A_{2}=0.5, A_{3}=0.25$, and $A_{4}=0.125$ or (b) $A_{2}=0.2, A_{3}=0.6$, and $A_{4}=0.4$. The phases of the fundamental and harmonics are chosen at random and the number of analyzed samples is $M=512$. The Hann window is adopted.

Fig. 3 shows the combined standard deviation of the frequency estimator achieved $\sigma_{\hat{\delta}}$, returned by simulations and (18) as a function of $v$ for the same signals as in the previous analysis and using the same simulations conditions. 


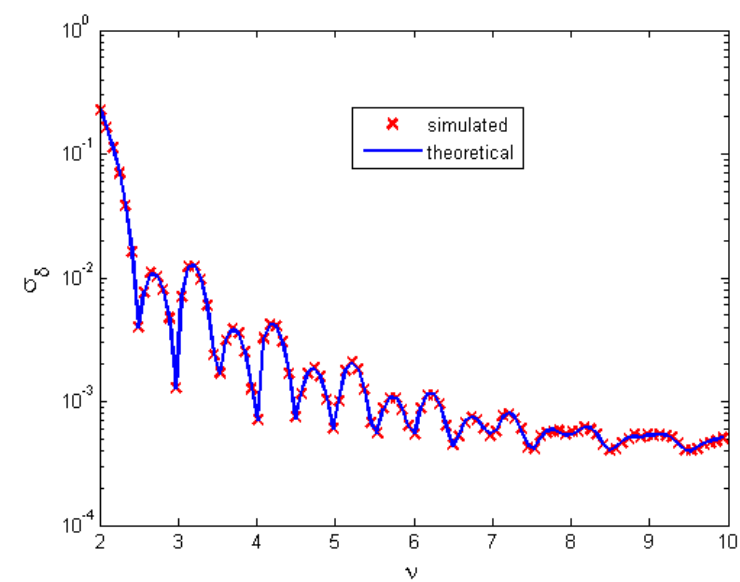

(a)

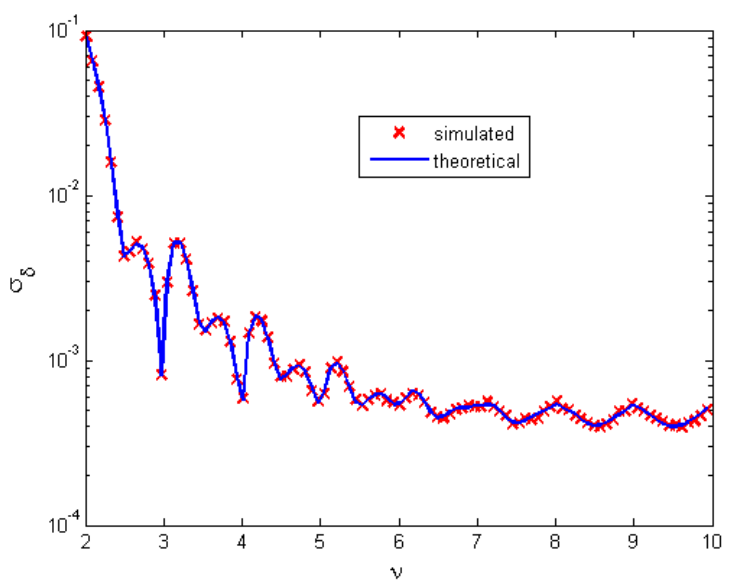

(b)

Fig. 3. Combined standard deviation of the frequency estimator versus $v$ returned by simulations (crosses) and (18) (solid lines) when analyzing a sine-wave with amplitude $A_{1}=1$ corrupted by 2nd, 3rd, and 4th harmonics with amplitudes (a) $A_{2}=0.5, A_{3}=0.25$, and $A_{4}=0.125$ or (b) $A_{2}=0.2, A_{3}=0.6$, and $A_{4}=0.4$. The phases of the fundamental and harmonics are chosen at random, the number of analyzed samples is $M$ $=512$, and $S N R=50 \mathrm{~dB}$. The Hann window is adopted.

Even in this case a very good agreement is achieved between simulation and theoretical results. Also, in Fig. 3(a) the combined standard deviation exhibits small oscillations when $v>8$ since the effect of wideband noise prevails on harmonics. The same behavior can be observed in Fig. 3(b) when $v>7$. Moreover, as we expected from the theoretical analysis, in the above situations the standard deviation exhibits maxima when coherent sampling occurs. Conversely, harmonics contribution prevails for small values of $v$, especially when $v<5$. However, this threshold decreases as SNR decreases.

The combined standard deviations of the frequency estimates returned by all the considered methods are compared in Fig. 4 considering the same noisy and harmonically distorted sine-waves as in Fig. 3. To analyze the influence of the number of harmonics $n_{h}$ in the MHSF method accuracy, the result obtained using either $n_{h}=2$ or 3 were analyzed. For each value of $v 1000$ runs were considered. 


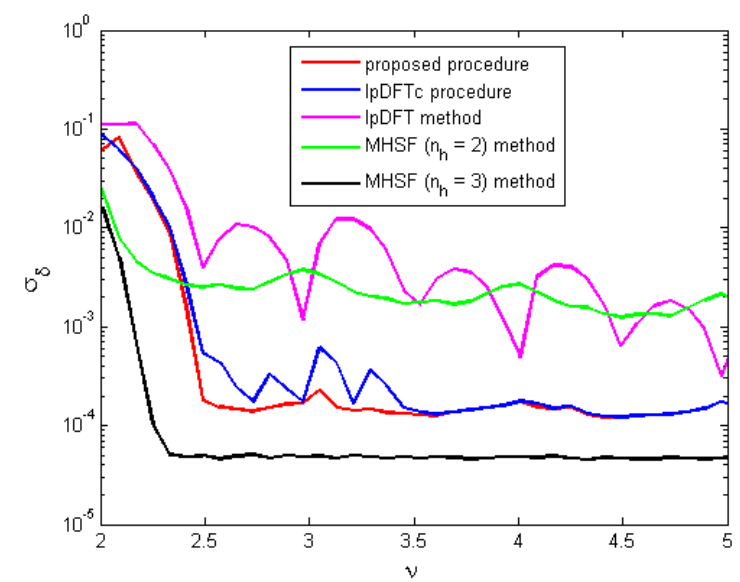

(a)

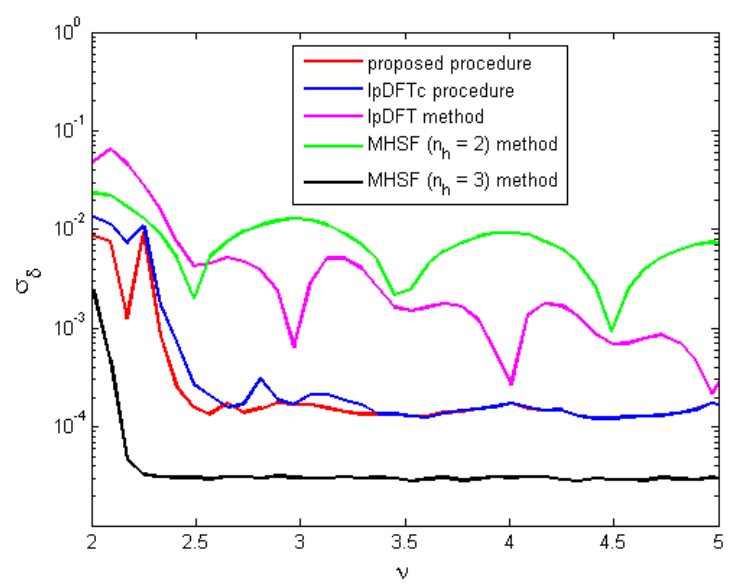

(b)

Fig. 4. Combined standard deviations of the frequency estimators versus $v$ returned by the classical IpDFT method, the proposed procedure $(\mu=10)$, the IpDFTc procedure, and the MHSF method with $n_{h}=$ 2 or 3, respectively. The signal parameters are: (a) $A_{1}=1, A_{2}=0.5, A_{3}=0.25$, and $A_{4}=0.125$ and (b) $A_{1}=$ $1, A_{2}=0.2, A_{3}=0.6$, and $A_{4}=0.4$. The phases of the fundamental and harmonics are chosen at random, the number of analyzed samples is $M=512$, and $S N R=50 \mathrm{~dB}$. The Hann window is adopted.

Fig. 4 shows that the proposed procedure outperforms the IpDFT method and exhibits better performance than the IpDFTc procedure in most situations when $v<3.5$, while almost the same performance are achieved for the remaining values of $v$ (i.e. $v \geq 3.5$ ) where the parameter estimates are accurate. Moreover, for $2.5<v<3.5$ the combined standard deviation related to the proposed method is almost constant and has a maximum when $\delta$ is close to 0 due to the behavior of the wideband noise effect, thus confirming that the harmonics have been effectively removed by the proposed procedure. Conversely, for $v>3.5$ both the proposed and the IpDFTc procedures have almost the same effectiveness in reducing harmonic contribution. Indeed, in these situations the frequency estimation accuracy is mainly limited by wideband noise. It is worth noticing that, unlike the IpDFTc procedure, the proposed one is based on the theoretical contribution of each harmonic to the frequency estimation error.

Many other simulations were performed for different values of harmonic amplitudes. It has been observed that the proposed procedure outperforms the IpDFTc one in most situations when the harmonic amplitudes are higher than $10 \%$ of the fundamental and $v<3.5$. Only, when the effect of the fundamental 
image component prevails over the harmonics one, the accuracy of the proposed procedure is almost equal to that ensured by the IpDFTc method when $v<3.5$.

As for the comparison with the MHSF method, the proposed procedure outperforms it when $n_{h}=2$, while it is less accurate when $n_{h}=3$. Indeed, the variance of this latter frequency estimator reaches the asymptotic Cramer-Rao lower bound [29] as soon as $v>2.5$ since no windowing is performed [26]. It is worth noticing that applying the MHSF method with $n_{h}>3$ no further accuracy improvement is achieved, despite a significant increase in the processing effort.

\subsection{Experimental results}

The accuracy of the proposed procedure was compared with the other considered state-of-the-art methods also through experimental results. In the experimental run, the harmonically distorted sine-waves are provided by an Agilent 33220A signal generator and acquired using a National Instruments 12-bit data acquisition board NI 6023. It should be noted that the adopted signal generator employs a 14-bit digital-toanalog converter (DAC) [32]. For sine-wave frequencies smaller than $20 \mathrm{kHz}$ the Total Harmonic Distortion ratio (THD) is smaller than $0.04 \%(-68 \mathrm{dBc})$ and the Spurious Free Dynamic Range (SFDR) is smaller than $-70 \mathrm{dBc}$ [32]. The NI-6023 acquisition board is equipped with a 12-bit successive approximations analog-to-digital converter (ADC) [33]. The maximum full-scale range and sampling frequency of the acquisition board are $20 \mathrm{~V}$ and $200 \mathrm{kHz}$, respectively [33]. The full-scale range and the sampling frequency were set to $10 \mathrm{~V}$ and $100 \mathrm{kHz}$, respectively. The generated sine-waves were affected by 2nd, 3rd, and 4th harmonics, and additive wideband noise. The fundamental component amplitude was set to $2 \mathrm{~V}$ and the signal frequency was varied in the range [497, 677] $\mathrm{Hz}$ with a step of $20 \mathrm{~Hz}$. For each frequency value, 1000 runs of $M=512$ samples each were considered. Thus, the number of acquired sinewave cycles is in the range $v \in(2.5,3.5)$, so that $l=3$. This range has been chosen to compare the performances of the considered methods when $v$ assumes small values, i.e. when the contribution of harmonics prevails over wideband noise. Two signals were generated, with 2nd and 3rd dominant 

estimated by the $\operatorname{MHSF}\left(n_{h}=3\right)$ method. These parameters and the Signal-to-Non-Harmonic Ratio (SNHR) [34] and THD of both these signals are provided in Table 1.

Table 1. Amplitudes of the fundamental component, significant harmonics, SNHR and THD for the two considered signals.

\begin{tabular}{|c|c|c|c|c|c|c|}
\hline Signal with: & $A_{1}[\mathrm{~V}]$ & $A_{2}[\mathrm{~V}]$ & $A_{3}[\mathrm{~V}]$ & $A_{4}[\mathrm{~V}]$ & $S N H R[\mathrm{dBc}]$ & $T H D[\%]$ \\
\hline dominant 2nd harmonic & 1.35 & 0.67 & 0.44 & 0.24 & 58 & 55 \\
\hline dominant 3rd harmonic & 1.11 & 0.22 & 0.71 & 0.62 & 58 & 75 \\
\hline
\end{tabular}

It is worth noticing that the inaccuracies of the signal generator are negligible as compared with the considered harmonic amplitudes. Moreover, since the acquisition board has a lower resolution than the signal generator DAC it is expected that wideband noise superimposed to the acquired signal is mainly

317 due to the acquisition.

318 The combined standard deviations of the achieved frequency estimates are shown in Fig. 5 as a 319 function of the number of observed cycles $v$, whose value was determined as the sample mean of the 320 estimates provides by the MHSF $\left(n_{h}=3\right)$ method.

321 Like in computer simulations, Fig. 5 shows that the proposed procedure outperforms the IpDFT and the $322 \operatorname{MHSF}\left(n_{h}=2\right)$ methods in all analyzed conditions, while the IpDFTc procedure is outperformed in most 323 analyzed conditions. However, the proposed procedure exhibits a lower accuracy than the MHSF method 324 based on the exact number of harmonics $n_{h}=3$. Also, curves in Figs. 4 and 5 show a very similar 325 behavior, so validating the performed computer simulations. 


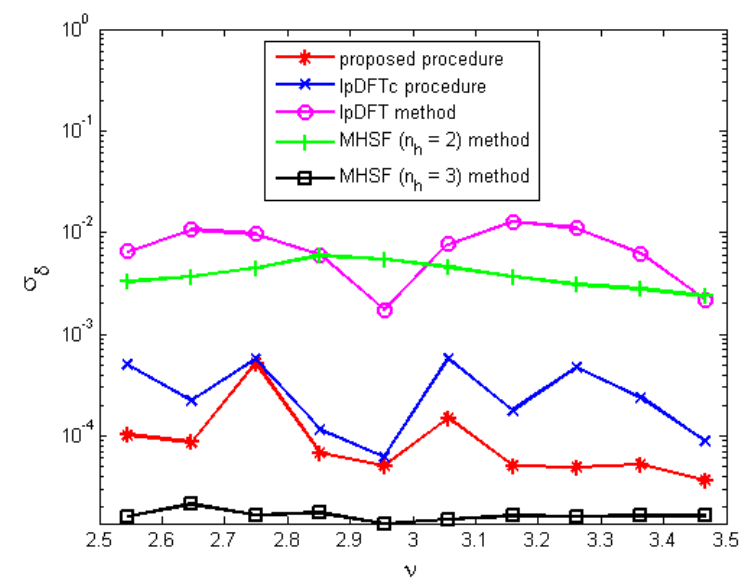

(a)

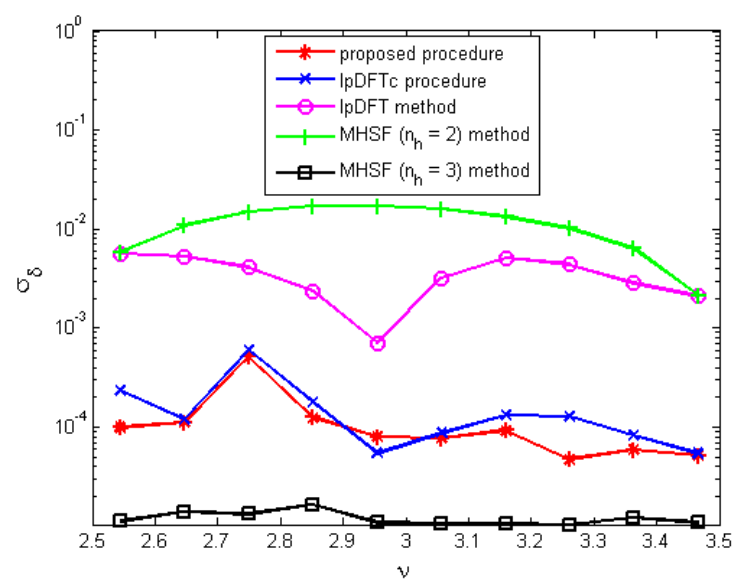

(b)

Fig. 5. Combined standard deviations of the frequency estimates versus $v$ returned by the proposed procedure, the IpDFTc procedure, the classical IpDFT method, and the MHSF method with $n_{h}=2$ or 3, respectively. Sine-wave corrupted by 2 nd, 3rd, and 4th harmonics with tones amplitudes: (a) $A_{1}=1.35, A_{2}$ $=0.67, A_{3}=0.44$, and $A_{4}=0.24 \mathrm{~V}$ or (b) $A_{1}=1.11, A_{2}=0.22, A_{3}=0.71$, and $A_{4}=0.62 \mathrm{~V}$. The number of analyzed samples is $M=512$ and the sampling frequency is $100 \mathrm{kHz}$. The Hann window is adopted.

\subsection{Computational complexity}

The processing times required by all the considered methods were also compared. A Matlab 7.1 environment running on a portable computer provided with a 2-GHz processor, 4G - RAM memory, and equipped with a Microsoft Windows 8.1 operating system was employed. The DFT samples were calculated directly, i.e. using (4) instead of the $f f(\cdot)$ function of Matlab. When considering the signals analyzed in Fig. 4, the average processing times over 1000 runs are given in Table 2.

Table 2. Average processing times over 1000 runs required by the considered methods.

\begin{tabular}{|c|c|}
\cline { 2 - 2 } \multicolumn{1}{c|}{} & $\begin{array}{c}\text { Average processing } \\
\text { time (ms) }\end{array}$ \\
\hline classical IpDFT method & 0.27 \\
\hline IpDFTc procedure & 0.44 \\
\hline proposed procedure & 0.94 \\
\hline MHSF method & 13.1 \\
\hline
\end{tabular}


Table 2 shows that the processing time required by the proposed method is about 2.1- and 3.5-times

higher than the IpDFTc procedure and classical IpDFT method, respectively. Conversely, the proposed procedure is 14-times faster than the MSHF method and this ratio is expected to increase when the number of significant harmonics is higher. In addition, to achieve accurate frequency estimates, the MHSF method needs the a-priori knowledge of the number of significant harmonics, so further increasing the processing effort. Thus, we can conclude that the proposed procedure can be advantageously used in the real-time applications requiring accurate frequency estimates.

\section{Conclusions}

In this paper the expressions for the frequency estimation error due to the spectral interference from the fundamental image component and harmonics and the estimator combined standard uncertainty due to the above disturbances and wideband noise have been derived when the MSD windows are adopted in the IpDFT method. The derived expressions allow us to determine the effect of each harmonic on the estimated frequency and, as a consequence, to identify which harmonics provide the most significant contribution. Based on the derived expressions a procedure capable to compensate all the significant effects of harmonics on the returned frequency estimates has been proposed. The accuracies of the derived expressions have been verified through computer simulations. It has been shown that the procedure proposed in this paper outperforms the classical IpDFT method and the IpDFTc procedure when few signal cycles are analyzed, i.e. when harmonics contribution dominates the effect of wideband noise. Moreover, despite the proposed procedure exhibits a lower accuracy than the MHSF method when applied on the exact (or higher) number of harmonics, it requires a much lower processing effort and so it can be advantageously employed in real-time high-accuracy applications. 


\section{Derivation of the analytical expression of the error $\Delta \delta$}

Using (5) and remembering that the window transform (6) is real-valued, it follows that:

$$
\begin{aligned}
\left|X_{w}(r)\right|^{2} & \cong \frac{1}{4}\left[A_{1}^{2} W^{2}(r-v)+A_{1}^{2} W^{2}(r+v)+\sum_{k=2}^{K} A_{k}^{2} W^{2}(r-k v)-2 A_{1}^{2} W(r-v) W(r+v) \cos \left(2 \varphi_{1}\right)\right. \\
& +2 \sum_{k=2}^{K} A_{1} A_{k} W(r-v) W(r-k v) \cos \left(\varphi_{1}-\varphi_{k}\right)-2 \sum_{k=2}^{K} A_{1} A_{k} W(r+v) W(r-k v) \cos \left(\varphi_{1}+\varphi_{k}\right) \\
& \left.+2 \sum_{k_{1}, k_{2}=2}^{K} A_{k_{1}} A_{k_{2}} W\left(r-k_{1} v\right) W\left(r-k_{2} v\right) \cos \left(\varphi_{k_{1}}-\varphi_{k_{2}}\right)\right] .
\end{aligned}
$$

Let's assume that the amplitude of the fundamental component is higher than harmonics and that the number of observed signal cycles $v$ is higher than the window's number of terms $H$. Thus, (6) shows that (A.1) due to the second, third, sixth, and seventh terms are negligible and:

$$
\begin{aligned}
\left|X_{w}(r)\right|^{2} & \cong \frac{1}{4}\left[A_{1}^{2} W^{2}(r-v)-2 A_{1}^{2} W(r-v) W(r+v) \cos \left(2 \varphi_{1}\right)\right. \\
& \left.+2 \sum_{k=2}^{K} A_{1} A_{k} W(r-v) W(r-k v) \cos \left(\varphi_{k}-\varphi_{1}\right)\right] .
\end{aligned}
$$

which can be written as:

$$
\left|X_{w}(r)\right|^{2} \cong \frac{1}{4} A_{1}^{2} W^{2}(r-v)\left[1-2 \frac{W(r+v)}{W(r-v)} \cos \left(2 \varphi_{1}\right)+2 \sum_{k=2}^{K} \frac{A_{k}}{A_{1}} \frac{W(r-k v)}{W(r-v)} \cos \left(\varphi_{k}-\varphi_{1}\right)\right] .
$$

377 Since $\left|\frac{W(r+v)}{W(r-v)}\right|<<1, \frac{A_{k}}{A_{1}}\left|\frac{W(r-k v)}{W(r-v)}\right|<<1,\left|\cos \left(2 \varphi_{1}\right)\right| \leq 1$, and $\left|\cos \left(\varphi_{k}-\varphi_{1}\right)\right| \leq 1$ it follows that

$\left|2 \frac{W(r+v)}{W(r-v)} \cos \left(2 \varphi_{1}\right)-2 \sum_{k=2}^{K} \frac{A_{k}}{A_{1}} \frac{W(r-k v)}{W(r-v)} \cos \left(\varphi_{k}-\varphi_{1}\right)\right|<<1$. By applying the first-order Taylor's series 


$$
\left|X_{w}(r)\right| \cong \frac{A_{1}}{2} W(r-v)-\frac{A_{1}}{2} W(r+v) \cos \left(2 \varphi_{1}\right)+\sum_{k=2}^{K} \frac{A_{k}}{2} W(r-k v) \cos \left(\varphi_{k}-\varphi_{1}\right)
$$

380 Using the substitutions $v=l+\delta$ and $r=l+p$, (A.4) becomes:

$$
\begin{array}{r}
\left|X_{w}(l+p)\right| \cong \frac{A_{1}}{2} W(-\delta+p)-\frac{A_{1}}{2} W(2 l+\delta+p) \cos \left(2 \varphi_{1}\right)+\sum_{k=2}^{K} \frac{A_{k}}{2} W((k-1) l+k \delta-p) \cos \left(\varphi_{k}-\varphi_{1}\right) \\
p=-1,0,1
\end{array}
$$

381 Since $\quad\left|\frac{W(2 l+\delta-1+s)}{W(-1-\delta+s)}\right|<<1 \quad$ and $\quad\left|\frac{A_{k}}{A_{1}} \frac{W((k-1) l+k \delta+1-s)}{W(-1-\delta+s)}\right|<<1 \quad$ we $\quad$ have $382\left|\frac{W(2 l+\delta-1+s)}{W(-1-\delta+s)} \cos \left(2 \varphi_{1}\right)-\sum_{k=2}^{K} \frac{A_{k}}{A_{1}} \frac{W((k-1) l+k \delta+1-s)}{W(-1-\delta+s)} \cos \left(\varphi_{k}-\varphi_{1}\right)\right|<<1$. From (9), and neglecting 383 that difference with respect to 1 , (A.5) provides:

$$
\alpha=\frac{\left|X_{w}(l+s)\right|}{\left|X_{w}(l-1+s)\right|} \cong \frac{W(-\delta+s)}{W(-1-\delta+s)}(1+\varepsilon),
$$

384 where:

$$
\begin{aligned}
\varepsilon & =-\left[\frac{W(2 l+\delta+s)}{W(-\delta+s)}-\frac{W(2 l+\delta-1+s)}{W(-1-\delta+s)}\right] \cos \left(2 \varphi_{1}\right) \\
& +\sum_{k=2}^{K} \frac{A_{k}}{A_{1}}\left[\frac{W((k-1) l+k \delta-s)}{W(-\delta+s)}-\frac{W((k-1) l+k \delta+1-s)}{W(-1-\delta+s)}\right] \cos \left(\varphi_{k}-\varphi_{1}\right)
\end{aligned}
$$

385 in which $s=0$ if $\left|X_{w}(l-1)\right|>\left|X_{w}(l+1)\right|$ and $s=1$ if $\left|X_{w}(l-1)\right|<\left|X_{w}(l+1)\right|$.

386 Using (8) the following equalities can be achieved:

$$
\begin{gathered}
W\left(1+(-1)^{s} \delta\right)=\frac{H-1-(-1)^{s} \delta}{H+(-1)^{s} \delta} W(\delta), \\
W\left(2 l+\delta+(-1)^{s+1}\right)=-\frac{2 l+\delta+(-1)^{s}(H-1)}{2 l+\delta+(-1)^{s+1} H} W(2 l+\delta), \\
W\left((k-1) l+k \delta+(-1)^{s}\right)=-\frac{(k-1) l+k \delta+(-1)^{s+1}(H-1)}{(k-1) l+k \delta+(-1)^{s} H} W((k-1) l+k \delta) .
\end{gathered}
$$

Using (A.8) - (A.10) after some algebra (A.7) becomes: 


$$
\begin{aligned}
\varepsilon & =\frac{(2 H-1)(l+\delta)}{\left(H-1+(-1)^{s+1} \delta\right) W(\delta)}\left[\frac{2(-1)^{s+1}}{2 l+\delta+(-1)^{s+1} H} W(2 l+\delta) \cos \left(2 \varphi_{1}\right)\right. \\
& \left.+\sum_{k=2}^{K} \frac{(-1)^{s}(k-1)}{(k-1) l+k \delta+(-1)^{s} H} \frac{A_{k}}{A_{1}} W((k-1) l+k \delta) \cos \left(\varphi_{k}-\varphi_{1}\right)\right] .
\end{aligned}
$$

388

Linearizing (11) we can write:

$$
\Delta \delta \cong \frac{\partial \hat{\delta}}{\partial \alpha} \Delta \alpha
$$

389 where $\Delta \alpha=\frac{\left|X_{w}(l+s)\right|}{\left|X_{w}(l-1+s)\right|}-\frac{W(-\delta+s)}{W(-1-\delta+s)}$. From (A.6) and (A.8) it follows that:

$$
\Delta \alpha \cong \frac{H+\delta-s}{H-\delta-1+s} \varepsilon,
$$

390

while from (11):

$$
\frac{\partial \hat{\delta}}{\partial \alpha}=\frac{(H-\delta-1+s)^{2}}{2 H-1} .
$$

By replacing (A.13) and (A.14) into (A.12) we achieve:

$$
\Delta \delta \cong \frac{(H+\delta-s)(H-\delta-1+s)}{2 H-1} \varepsilon .
$$

392

Finally, using (A.11), (A.15) becomes:

$$
\begin{aligned}
\Delta \delta \cong & \frac{\left(H+(-1)^{s} \delta\right)(l+\delta)}{W(\delta)}\left[\frac{2(-1)^{s+1}}{2 l+\delta+(-1)^{s+1} H} W(2 l+\delta) \cos \left(2 \varphi_{1}\right)\right. \\
& \left.+\sum_{k=2}^{K} \frac{(k-1)(-1)^{s}}{(k-1) l+k \delta+(-1)^{s} H} \frac{A_{k}}{A_{1}} W((k-1) l+k \delta) \cos \left(\varphi_{k}-\varphi_{1}\right)\right]
\end{aligned}
$$


[1] S.L. Marple, Digital spectral analysis (Prentice-Hall, Englewood Cliffs, 1987).

[2] D. Petri, D. Belega, D. Dallet, ch. 10 - Dynamic testing of analog-to-digital converters by means of the sine-fitting algorithms, pp. 309-340, Design, Modeling and Testing of Data Converters (Springer Berlin Heidelberg, Germany, 2014).

[3] I. Santamaria, C. Pantaleon, J. Ibanez, A comparative study of high-accuracy frequency estimation methods, Mech. Syst. Signal Process. 14(2000) 819-834.

[4] I. Santamaria-Caballerro, C.J. Panteleon-Prieto, J. Ibanez-Diaz, Improved procedures for estimating amplitudes and phases of harmonics with applications to vibration analysis, IEEE Trans. Instrum. Meas. 47 (1) (1998) 209-214.

[5] D.C. Rife, G.A. Vincent, Use of the discrete Fourier transform in the measurement of frequencies and levels of tones, Bell Syst. Tech. J. 49 (1970) 197-228.

[6] T. Grandke, Interpolation algorithms for discrete Fourier transforms of weighted signals, IEEE Trans. Instrum. Meas. 32 (2) (1983) 350-355.

[7] C. Offelli, D. Petri, The influence of windowing on the accuracy of multifrequency signal parameter estimation, IEEE Trans. Instrum. Meas. 41 (2) (1992) 256-261.

[8] C. Offelli, D. Petri, Interpolation techniques for real-time multifrequency analysis, IEEE Trans. Instrum. Meas. 39 (1) (1990) 106-111.

[9] D. Belega, D. Dallet, Multifrequency signal analysis by interpolated DFT method with maximum sidelobe decay windows, Measurement 42 (3) (2009) 420-426.

[10] D. Belega, D. Dallet, D. Petri, Statistical description of the sine-wave frequency estimator provided by the interpolated DFT method, Measurement 45 (1) (2012) 109-117.

[11] K. Ding, M. Xie, X.F. Zhang, Phase difference correction method for phase and frequency in spectral analysis, Mech. Syst. Signal Process. 14 (2000) 835-843.

[12] Y. Huang, K. Xu, Study on the spectrum correcting method based on phase difference, Journal of Vibration and Shock 24 (2005) 77-79. 
[13] L.-M. Zhu, H.-X Li, H. Ding, Y.-L. Xiong. Noise influence on estimation of signal parameter from the phase difference of discrete Fourier transforms, Mech. Syst. Signal Process. 16(2002) 991-1004.

[14] L.-M. Shu, H.-X. Li, H. Ding, Estimation of multi-frequency signal parameters by frequency domain nonlinear least squares, Mech. Syst. Signal Process. 19(2005) 955-973.

[15] P. Carbone, E. Nunzi, D. Petri, A frequency-domain-based least-squares estimation of multifrequency signal parameters, IEEE Trans. Instrum. Meas. 49 (3) (2000) 555-558.

[16] H.B. Lin, K. Ding, Energy based signal parameter estimation method and a comparative study of different frequency estimators, Mech. Syst. Signal Process. 25 (2011) 454-464.

[17] D. Belega, D. Dallet, D. Petri, Accuracy of the normalized frequency estimation of a discrete-time sinewave by the energy-based method, IEEE Trans. Instrum. Meas. 61 (1) (2012) 111-121.

[18] A.H. Nuttall, Some windows with very good sidelobe behavior, IEEE Trans. Acoust., Speech, Signal Process. ASSP-29 (1) (1981) 84-91.

[19] D. Agrež, Dynamics of frequency estimation in the frequency domain, IEEE Trans. Instrum. Meas. 56 (6) (2007) 2111-2118.

[20] D. Belega, D. Dallet, D. Petri, Accuracy of sine-wave frequency estimation by multipoint interpolated DFT approach, IEEE Trans. Instrum. Meas. 59 (11) (2010) 2808-2815.

[21] J. Borkowski, D. Kania, L. Mroczka, Interpolated-DFT-based fast and accurate frequency estimation for the control of power, IEEE Trans. Ind. Electron. 61(12) (2014) 7026-7034.

[22] C. Liguori, A. Paolillo, A. Pignotti, An intelligent FFT-analyzer with harmonic interference effect correction and uncertainty evaluation, in Proc. Instrumentation and Measurement Technology Conference (IMTC), vol. 2, pp. 987-992, Vail, Colorado, USA, May 20-22, 2003.

[23] C. Liguori, A. Paolillo, A. Pignotti, Estimation of signal parameters in the frequency domain in the presence of harmonic interference: a comparative analysis, IEEE Trans. Instrum. Meas. 55 (2) (2006) 562569.

[24] P.M. Ramos, M.F.da Silva, R.C. Martins, A.M. Cruz Serra, Simulation and experimental results of multiharmonic least-squares fitting algorithms applied to periodic signals, IEEE Trans. Instrum. Meas. 55 (2) (2006) 646-651. 
[25] A. Ferrero, R. Ottoboni, High-accuracy Fourier analysis based on synchronous sampling techniques, IEEE Trans. Instrum. Meas. 41 (6) (1992) 780-785.

[26] F.J. Harris, On the use of windows for harmonic analysis with the discrete Fourier transform, Proceedings of the IEEE 66 (1) (1978) 51-83.

[27] S. Gori, C. Narduzzi, Application of a phase measurement algorithm to digitizing oscilloscope characterization, IEEE Trans. Instrum. Meas. 49 (6) (2000) 1211- 1215.

[28] D. Belega, D. Petri, Accuracy analysis of the multicycle synchrophasor estimator provided by the interpolated DFT algorithm, IEEE Trans. Instrum. Meas. 62 (5) (2013) 942- 953.

[29] D.C. Rife, R.R. Boorstyn, Single tone parameter estimation from discrete-time observations, IEEE Trans. Inf. Theory, IT-20 (5) (1974) 591-598.

[30] S.M. Kay, Fundamentals of Statistical Signal Processing: Estimation Theory (Prentice-Hall, Upper Saddle River, NJ, 1993).

[31] Guide for the expression of uncertainty in measurement (GUM), International Organization for Standardization, Switzerland, second edition, 1995.

[32] Agilent 33220A 20 MHz Function/Arbitrary Waveform Generator, User’s Guide, Agilent, 2007.

[33] NI6023E/6024E/6025E Family Specifications, National Instruments, 2005.

[34] IEEE Std. 1241, Standard for Terminology and Test Methods for Analog-to-Digital Converters, 2010. 